\title{
Estimation and Control for Systems with Nonlinearly Parameterized Perturbations *
}

\author{
Håvard Fjær Grip ${ }^{*, * *}$ Tor A. Johansen ${ }^{*}$ Lars Imsland ${ }^{* *}$ \\ * NTNU, Department of Engineering Cybernetics, NO-7491 \\ Trondheim, Norway (e-mail: grip@itk.ntnu.no) \\ ** SINTEF ICT, Applied Cybernetics, NO-7465 Trondheim, Norway
}

\begin{abstract}
A class of systems influenced by nonlinearly parameterized perturbations is considered. An estimation scheme is developed whereby exponentially stable estimates of the unknown parameters can be obtained with an arbitrarily large region of attraction, provided the states are available for measurement. The method applies to a class of perturbations with the property that an exponentially stable estimate of the unknown parameters can be obtained if the whole perturbation is known. Compensation for the perturbations in the system equations is considered for a class of systems which have uniformly globally bounded solutions and for which the origin is globally asymptotically stable when no perturbations are present. Examples with simulations are given in order to illustrate the results.
\end{abstract}

Keywords: Nonlinear system control; Adaptive control; Nonlinear observer and filter design

\section{INTRODUCTION}

An important issue in control applications is the handling of unknown perturbations to system equations. Such perturbations can be the result of external disturbances or internal plant changes, such as a configuration change, system fault or changes in physical plant characteristics. Frequently, such perturbations can be characterized in terms of a vector of unknown, constant parameters.

Adaptive control techniques aim to counteract such perturbations by introducing estimates of the unknown parameters and using these to cancel the effect of the perturbations. When the perturbations are linear in the unknown parameters, design of adaptive control schemes is often straightforward, and techniques for handling such cases are well developed (see, e.g., Krstić et al. (1995); Ioannou and Sun (1996)). In the case of nonlinearly parameterized perturbations, the problem is naturally more complicated, and the range of available design techniques is more limited. One approach is to use a gradient algorithm, as in linearly parameterized systems, which may yield poor results or instability for nonlinear parameterizations. Another common approach is over-parameterization, whereby extra unknown parameters are introduced in order to express the perturbation as linear in the parameters. This increases complexity and may affect performance by reducing the convergence rate of the parameter estimates or introducing stricter persistency of excitation conditions.

To address the problem of nonlinearly parameterized perturbations, some techniques have been introduced which do not resort to approximations. In Fomin et al. (1981); Ortega (1996), stability is proven for a gradient-type approach when the nonlinear parameterization is concave. In Annaswamy et al. (1998), the convexity or concavity of some parameterizations is exploited by introducing a tuning function and adaptation based on a min-max optimization strategy, thus achieving tracking of the controlled variables to within a desired precision. The approach is extended to more general nonlinear parameterizations in Loh et al. (1999).

Other results, such as Bošković (1995, 1998); Zhang et al. (2000), have focused on first-order systems with certain fractional parameterizations, proving convergence of the controlled state, but without studying convergence of the parameter estimates. In $\mathrm{Qu}$ (2003), an estimation-based approach is introduced for a class of higher-order systems with a matrix fractional parameterization. Here, an auxiliary estimate of the full perturbation is introduced, which is used in the estimation of the unknown parameters. The method achieves global boundedness and ultimate boundedness to within a desired precision. In $\mathrm{Qu}$ et al. (2006), yet another approach is presented for more general nonlinear parameterizations, where, instead of relying on the certainty equivalence principle, the parameter estimate used in the control law is biased by an appropriately chosen vector function. Conditions are given under which the errors in both the controlled states and the estimated parameters converge to zero.

Another way of dealing with undesired perturbations can be found in Chakrabortty and Arcak (2007), where the whole perturbation is estimated using a high-gain approach. This estimate is then used to counteract the perturbation. For the systems considered therein, this can be done arbitrarily fast, and the performance of the unperturbed system can therefore be recovered. The approach considered in this paper has similarities to Chakrabortty and Arcak (2007), but it also exploits available structural information by estimating an unknown parameter vector in addition to the full perturbation. This has certain advantages, such as being able to handle highly time-varying

\footnotetext{
* This research is supported by the Research Council of Norway.
} 
perturbations, and avoiding some of the problems related to noise that are inherent in high-gain designs.

The results presented here assumes a relatively small class of perturbations, among other things because the perturbations are required to be persistently exciting. For those perturbations that can be handled, however, the method is often highly effective, especially with respect to providing fast parameter estimates. This may be useful not only for direct compensation, but also as part of other control schemes where fast parameter estimates are required.

\section{NOTATION AND DEFINITIONS}

Conventional notation is used to denote estimates and error variables, meaning that for some quantity $z, \hat{z}$ represents its estimate and $\tilde{z}=z-\hat{z}$ is an error variable. For a vector $z, z_{i}$ denotes its $i$ 'th element. The norm operator $\|\cdot\|$ denotes the Euclidian norm for vectors and the induced Euclidian norm for matrices. The minimum eigenvalue of a matrix $A$ is denoted $\lambda_{\min }(A)$. We denote by $\mathbb{R}_{\geq 0}$ and $\mathbb{R}_{>0}$ the non-negative and the positive real numbers. For two sets $E, F \subset \mathbb{R}^{n}$, we write $(E-F):=$ $\left\{z_{1}-z_{2} \in \mathbb{R}^{n} \mid z_{1} \in E, z_{2} \in F\right\}$. Throughout this paper, when considering systems on the form

$$
\dot{z}=F(t, z),
$$

we implicitly assume that $F: \mathbb{R}_{\geq 0} \times \mathbb{R}^{n} \rightarrow \mathbb{R}^{n}$ is piecewise continuous in $t$ and locally Lipschitz continuous in $z$, uniformly in $t$, on $\mathbb{R}_{\geq 0} \times \mathbb{R}^{n}$. We denote by $z(t)$ the solution of (1), initialized at time $t=t_{0}$ with initial condition $z=z\left(t_{0}\right)$. In this paper, we make use of the abbreviations UGB (uniform global boundedness) and UGAS (uniform global asymptotic stability). For definitions of these and related terms, we refer to Khalil (2002).

\section{PROBLEM FORMULATION}

Starting with any general system, we consider systems which, by the appropriate state transformations and choice of control law, can be expressed in the following form:

$$
\dot{x}=f(t, x)+B(t, x)(g(t, x, \theta)+v(t, x)),
$$

where $x \in \mathbb{R}^{n}$ are measured states and $\theta \in \mathbb{R}^{p}$ is a vector of unknown, constant parameters. The functions $f: \mathbb{R}_{\geq 0} \times \mathbb{R}^{n} \rightarrow \mathbb{R}^{n}, B: \mathbb{R}_{\geq 0} \times \mathbb{R}^{n} \rightarrow \mathbb{R}^{n \times m}$ and $v: \mathbb{R}_{\geq 0} \times$ $\mathbb{R}^{n} \rightarrow \mathbb{R}^{m}$ can be evaluated from available measurements, and $g: \mathbb{R}_{\geq 0} \times \mathbb{R}^{n} \times \mathbb{R}^{p} \rightarrow \mathbb{R}^{m}$ is differentiable with respect to $\theta$ and can be evaluated if $\theta$ is known.

We first consider the problem of designing an estimator for the unknown parameter vector $\theta$. We then consider the case when available control inputs can be used to create a system transformable to the form of (2) with $v(t, x)=-g(t, x, \hat{\theta})$, where $\hat{\theta}$ represents a parameter estimate. We derive stability results for this system based on the convergence properties of the parameter estimation error, which is defined as $\tilde{\theta}:=\theta-\hat{\theta}$.

\subsection{Restriction of Parameters}

In most practical circumstances, it is known from physical considerations that $\theta$ is restricted to some bounded set of values. This is a significant advantage when it comes to satisfying the assumptions made later in this paper. To simplify the exposition, we therefore assume throughout this paper that the set of possible parameters is indeed bounded. In designing update laws for parameter estimates, we will also assume that a parameter projection can be implemented in a manner similar to that described in Krstić et al. (1995), restricting the parameter estimates to a compact, convex set $\Theta \subset \mathbb{R}^{p}$ (defined slightly larger than the set of possible parameter values). For the sake of brevity, we shall include the projection in the examples given throughout the paper only as a generic additive term p.t. in the differential equations, and largely ignore the term in the following analysis. We only note here that the analysis is compatible with the use of a projection term, with only minor modifications (e.g., to bounds and constants).

\section{PARAMETER ESTIMATION}

In this section, we present a method for estimating the unknown parameter vector $\theta$ when $x(t)$ is bounded. For ease of notation, we introduce $\phi:=B(t, x) g(t, x, \theta)$, which represents the full unknown perturbation in (2).

\subsection{Estimation of $\theta$ from $\phi$}

The estimation scheme is based on generating an estimate of $\phi$. This estimate will be denoted $\hat{\phi}$, and it will in turn be used to estimate $\theta$. For this to work, there needs to exist an update law

$$
\dot{\hat{\theta}}=u_{\theta}(t, x, \hat{\phi}, \hat{\theta}),
$$

which, if $\phi$ were known (i.e., $\hat{\phi}=\phi$ ), would provide an unbiased asymptotic estimate of $\theta$. This is the subject of the following assumption. We emphasize that we do not assume that $\phi$ is in fact known.

Assumption 1. For each compact set $K \subset \mathbb{R}^{n}$, there exists a continuously differentiable function $V_{\mathrm{u}}: \mathbb{R}_{\geq 0} \times(\Theta-\Theta) \rightarrow$ $\mathbb{R}_{\geq 0}$ and positive constants $a_{1}, a_{2}, a_{3}$ and $a_{4}$ such that for all $(t, x, \phi, \hat{\theta}) \in \mathbb{R}_{\geq 0} \times K \times \mathbb{R}^{n} \times \Theta$,

$$
\begin{gathered}
a_{1}\|\tilde{\theta}\|^{2} \leq V_{\mathrm{u}}(t, \tilde{\theta}) \leq a_{2}\|\tilde{\theta}\|^{2}, \\
\frac{\partial V_{\mathrm{u}}}{\partial t}(t, \tilde{\theta})-\frac{\partial V_{\mathrm{u}}}{\partial \tilde{\theta}}(t, \tilde{\theta}) u_{\theta}(t, x, \phi, \hat{\theta}) \leq-a_{3}\|\tilde{\theta}\|^{2}, \\
\left\|\frac{\partial V_{\mathrm{u}}}{\partial \tilde{\theta}}(t, \tilde{\theta})\right\| \leq a_{4}\|\tilde{\theta}\| .
\end{gathered}
$$

Furthermore, the update law (3) ensures that if $\hat{\theta}\left(t_{0}\right) \in \Theta$, then for all $t \geq t_{0}, \hat{\theta}(t) \in \Theta$.

Satisfying Assumption 1 Assumption 1 guarantees that the origin of the error dynamics

$$
\dot{\tilde{\theta}}=-u_{\theta}(t, x, \phi, \theta-\tilde{\theta}),
$$

which occurs if $\hat{\phi}=\phi$, is uniformly exponentially stable with $(\Theta-\Theta)$ contained in the region of attraction. In essence, this amounts to being able to solve the inverse problem of finding $\theta$ given $\phi=B(t, x) g(t, x, \theta)$, using some numerical method which can be described as a differential equation for $\hat{\theta}$. We will not state exact conditions for when it is possible to satisfy this assumption. Typically, however, the idea is to look for some function $M(t, x)$ which is such that

$$
\tilde{\theta}^{\top} M(t, x) B(t, x)(g(t, x, \theta)-g(t, x, \hat{\theta})) \geq \tilde{\theta}^{\top} P(t, x) \tilde{\theta},
$$


where $P(t, x)$ is positive semidefinite, and to use an update function similar to

$$
u_{\theta}(t, x, \phi, \hat{\theta})=k_{\theta} M(t, x)(\phi-B(t, x) g(t, x, \hat{\theta}))+\text { p.t. }
$$

where $k_{\theta}$ is a positive scalar gain. The types of perturbations for which this is possible can be described as monotonic in a generalized sense.

If $P(t, x)$ is positive definite (uniformly in $t$ ), it is immediately clear that (8) satisfies Assumption 1, using the Lyapunov function $V_{\mathrm{u}}(t, \tilde{\theta})=\frac{1}{2} \tilde{\theta}^{\top} \tilde{\theta}$ (and assuming that the projection term does not contribute in a positive direction in the time derivative). The following example illustrates this case.

Example 1. Consider the perturbation $B(t, x) g(t, x, \theta)=$ $g(\theta)=\left[\theta_{1}, \theta_{1}^{2}+\theta_{2}\right]^{\top}$, with $\Theta=[-10,10] \times[-10,10]$. Using the mean value theorem, we find that

$$
g(\theta)-g(\hat{\theta})=\left[\begin{array}{cc}
1 & 0 \\
2 \bar{\theta}_{1} & 1
\end{array}\right] \tilde{\theta}
$$

where $\bar{\theta}_{1}$ is a value between $\theta_{1}$ and $\hat{\theta}_{1}$. Selecting $M(t, x)=$ $M=\operatorname{diag}\left(K_{M}, 1\right)$ therefore yields

$$
\tilde{\theta}^{\top} M(g(\theta)-g(\hat{\theta}))=\tilde{\theta}^{\top}\left[\begin{array}{cc}
K_{M} & 0 \\
2 \bar{\theta}_{1} & 1
\end{array}\right] \tilde{\theta} .
$$

Using the fact that $\left|\bar{\theta}_{1}\right|<10$ (because both $\theta$ and $\hat{\theta}$ are contained in $\Theta$ ), it is easily confirmed that the matrix in the above expression is positive definite if $K_{M}$ is chosen sufficiently large.

If $P(t, x)$ is positive definite as in Example 1, it implies that the inversion problem can be solved arbitrarily fast by increasing the gain in (8). In many cases, this is not possible, but it may still be possible to satisfy Assumption 1 via a positive semidefinite $P(t, x)$. This case is more complicated, as it is necessary to investigate whether $P(t, x)$ is persistently exciting; that is, whether there exist $T>0$ and $\varepsilon>0$ such that for all $t \in \mathbb{R}_{\geq 0}$,

$$
\int_{t}^{t+T} P(\tau, x(\tau)) \mathrm{d} \tau \geq \varepsilon I>0 .
$$

If this inequality holds, it is often possible to use a Lyapunov function candidate (LFC) on the following form:

$$
V_{\mathrm{u}}(t, \tilde{\theta})=\frac{1}{2} \tilde{\theta}^{\top} \tilde{\theta}-\mu \tilde{\theta}^{\top} \int_{t}^{\infty} \mathrm{e}^{t-\tau} P(\tau, x(\tau)) \mathrm{d} \tau \tilde{\theta},
$$

where $\mu>0$ is a small constant. This is illustrated by the following example.

Example 2. Consider the perturbation $B(t, x) g(t, x, \theta)=$ $g(t, \theta)=\sin (t)\left[\theta_{1}, \theta_{1}^{2}+\theta_{2}\right]^{\top}$, with $\Theta=[-10,10] \times[-10,10]$. Using the same argument as in the last example, we see that by selecting $M(t, x)=M(t)=\sin (t) \operatorname{diag}\left(K_{M}, 1\right)$ and choosing $K_{M}$ sufficiently large, (7) is satisfied with $P(t, x)=P(t)=c \sin ^{2}(t)$ for some constant $c>0$. Moreover, with $T$ chosen as any positive number, we have $\int_{t}^{t+T} \sin ^{2}(\tau) \mathrm{d} \tau \geq \varepsilon>0$ (uniformly in $t$, for some number $\varepsilon)$. We therefore use $M(t)$ in the update function (8), and the $\operatorname{LFC}$ from (10). We first note that $\left(\frac{1}{2}-c \mu\right)\|\tilde{\theta}\|^{2} \leq$ $V_{\mathrm{u}}(t, \tilde{\theta}) \leq \frac{1}{2}\|\tilde{\theta}\|^{2}$. Hence, $V_{\mathrm{u}}$ is positive definite if we choose $\mu<1 /(2 c)$. Taking the time derivative (not considering the projection term) yields

$$
\begin{aligned}
\dot{V}_{\mathrm{u}}(t, \tilde{\theta}) \leq & -\left(1-2 c \mu \int_{t}^{\infty} \mathrm{e}^{t-\tau} \sin ^{2}(\tau) \mathrm{d} \tau\right) \\
& \cdot k_{\theta} \tilde{\theta}^{\top} M(t)(g(t, \theta)-g(t, \hat{\theta})) \\
& +c \mu \sin ^{2}(t) \tilde{\theta}^{\top} \tilde{\theta}-c \mu \int_{t}^{\infty} \mathrm{e}^{t-\tau} \sin ^{2}(\tau) \mathrm{d} \tau \tilde{\theta}^{\top} \tilde{\theta} \\
\leq & -\left(k_{\theta}-2 k_{\theta} c \mu-\mu\right) c \sin ^{2}(t)\|\tilde{\theta}\|^{2}-c \mu \mathrm{e}^{-T} \varepsilon\|\tilde{\theta}\|^{2} .
\end{aligned}
$$

It follows that the time derivative is negative definite provided $\mu<k_{\theta} /\left(1+2 c k_{\theta}\right)$.

\subsection{Estimator}

We now introduce the full estimator for the unknown parameter vector:

$$
\begin{aligned}
& \dot{z}=-K_{\phi}(f(t, x)+B(t, x) v(t, x)+\hat{\phi}) \\
&-B(t, x) \frac{\partial g}{\partial \theta}(t, x, \hat{\theta}) u_{\theta}(t, x, \hat{\phi}, \hat{\theta}), \\
& \hat{\phi}=z+K_{\phi} x+B(t, x) g(t, x, \hat{\theta}), \\
& \dot{\hat{\theta}}=u_{\theta}(t, x, \hat{\phi}, \hat{\theta}),
\end{aligned}
$$

where $K_{\phi}$ is a positive definite gain matrix. The full estimator can be considered to consist of two parts: one is an estimate of $\phi$ described by (11a), (11b), and the other is the update law from Section 4.1, where the perturbation $\phi$ has been replaced with the estimate $\hat{\phi}$.

In order to study the properties of the estimator, we consider the dynamics of the errors $\tilde{\phi}$ and $\tilde{\theta}$. Taking the time derivative of $\tilde{\phi}=\phi-\hat{\phi}$, we may write

$$
\begin{aligned}
\dot{\tilde{\phi}}= & \frac{\partial}{\partial t}(B(t, x) g(t, x, \theta))+\frac{\partial}{\partial x}(B(t, x) g(t, x, \theta)) \dot{x} \\
& +K_{\phi}(f(t, x)+B(t, x) v(t, x)+\hat{\phi}) \\
& +B(t, x) \frac{\partial g}{\partial \theta}(t, x, \hat{\theta}) u_{\theta}(t, x, \hat{\phi}, \hat{\theta})-K_{\phi} \dot{x} \\
& -\frac{\partial}{\partial t}(B(t, x) g(t, x, \hat{\theta}))-\frac{\partial}{\partial x}(B(t, x) g(t, x, \hat{\theta})) \dot{x} \\
& -B(t, x) \frac{\partial g}{\partial \theta}(t, x, \hat{\theta}) u_{\theta}(t, x, \hat{\phi}, \hat{\theta}) .
\end{aligned}
$$

At this point, it is convenient to define the function

$$
\begin{aligned}
d(t, x, \tilde{\theta}):= & \frac{\partial}{\partial t}(B(t, x) g(t, x, \theta)-B(t, x) g(t, x, \hat{\theta})) \\
& +\frac{\partial}{\partial x}(B(t, x) g(t, x, \theta)-B(t, x) g(t, x, \hat{\theta})) \dot{x} .
\end{aligned}
$$

This can be seen as the time derivative of $B(t, x)(g(t, x, \theta)-$ $g(t, x, \hat{\theta}))$ when $\hat{\theta}$ is kept constant. Using this function and the fact that $\dot{x}-f(t, x)-B(t, x) v(t, x)=\phi$, we may rewrite the above expression and write the error dynamics of the estimator as

$$
\begin{aligned}
\dot{\tilde{\phi}} & =-K_{\phi} \tilde{\phi}+d(t, x, \tilde{\theta}), \\
\dot{\tilde{\theta}} & =-u_{\theta}(t, x, \phi, \hat{\theta})+\left(u_{\theta}(t, x, \phi, \hat{\theta})-u_{\theta}(t, x, \hat{\phi}, \hat{\theta})\right) .
\end{aligned}
$$

For convenience, we define the error variable $\xi:=\left[\tilde{\phi}^{\top}, \tilde{\theta}^{\top}\right]^{\top}$ and the set $\Xi:=\mathbb{R}^{n} \times(\Theta-\Theta)$.

To state our results on the estimation of $\theta$, we need some further assumptions.

Assumption 2. For all $(t, x, \tilde{\theta}) \in \mathbb{R}_{\geq 0} \times \mathbb{R}^{n} \times(\Theta-\Theta)$, the function $d(t, x, \tilde{\theta})$ is well-defined, and for each compact set $K \subset \mathbb{R}^{n}$, there exist numbers $L_{1}>0$ and $L_{2}>0$ such that 
for all $(t, x, \tilde{\theta}) \in \mathbb{R}_{\geq 0} \times K \times(\Theta-\Theta),\|d(t, x, \tilde{\theta})\| \leq L_{1}\|\tilde{\theta}\|$; and for all $(t, x, \phi, \hat{\phi}, \hat{\theta}) \in \mathbb{R}_{>0} \times K \times \mathbb{R}^{n} \times \mathbb{R}^{n} \times \Theta$, $\|u(t, x, \phi, \hat{\theta})-u(t, x, \hat{\phi}, \hat{\theta})\| \leq L_{2}\|\tilde{\phi}\|$.

The next lemma states the basic result on estimation of $\theta$. Lemma 1. Suppose Assumptions 1 and 2 hold and that for all $t \in \mathbb{R}_{>0},\|x(t)\|$ is uniformly bounded. Then there exists $k^{\prime}>0$ such that if $K_{\phi}$ is chosen such that $\lambda_{\min }\left(K_{\phi}\right)>k^{\prime}$, then the origin of (12) is uniformly exponentially stable with $\Xi$ contained in the region of attraction.

Proof. Assumption 1 ensures that if $\hat{\theta}\left(t_{0}\right) \in \Theta$, then $\hat{\theta}(t) \in \Theta$. It follows that no trajectory of the estimator error dynamics can escape $\Xi$. The uniform boundedness of $x(t)$ implies that $x(t) \in K$, where $K \subset \mathbb{R}^{n}$ is compact. We may therefore make use of the inequalities from Assumptions 1 and 2 corresponding to $x(t) \in K$. Define the LFC $V_{\mathrm{p}}(t, \xi):=V_{\mathrm{u}}(t, \tilde{\theta})+\frac{1}{2} \tilde{\phi}^{\top} \tilde{\phi}$, where $V_{\mathrm{u}}$ is the Lyapunov function from Assumption 1. We investigate its time derivative on $\Xi$. Using the inequalities from Assumptions 1 and 2, we may write

$$
\dot{V}_{\mathrm{p}}(t, \xi) \leq-[\|\tilde{\phi}\|\|\tilde{\theta}\|] Q\left[\begin{array}{c}
\|\tilde{\phi}\| \\
\|\tilde{\theta}\|
\end{array}\right],
$$

where

$$
Q=\left[\begin{array}{cc}
\lambda_{\min }\left(K_{\phi}\right) & -\frac{1}{2}\left(a_{4} L_{2}+L_{1}\right) \\
-\frac{1}{2}\left(a_{4} L_{2}+L_{1}\right) & a_{3}
\end{array}\right]
$$

The matrix $Q$ is positive definite provided its leading principal minors are positive. The first-order leading principal minor is $\lambda_{\min }\left(K_{\phi}\right)>0$ and the second-order leading principal minor, or determinant, is $a_{3} \lambda_{\min }\left(K_{\phi}\right)-\frac{1}{4}\left(a_{4} L_{2}+\right.$ $\left.L_{1}\right)^{2}$, which is made positive by choosing $\lambda_{\min }\left(K_{\phi}\right)>k^{\prime}:=$ $\left(a_{4} L_{2}+L_{1}\right)^{2} /\left(4 a_{3}\right)$. We therefore have

$$
\dot{V}_{\mathrm{p}}(t, \xi) \leq-\lambda_{\min }(Q)\|\xi\|^{2} \leq-\left(\lambda_{\min }(Q) / \bar{c}\right) V_{\mathrm{p}}(t, \xi),
$$

where $\bar{c}:=\max \left\{a_{2}, \frac{1}{2}\right\}$. As in the proof of Khalil (2002, Th. 4.10 ), we may now invoke the comparison lemma to prove that there exist positive constants $k_{\mathrm{e}}$ and $\lambda$ such that for all $\xi\left(t_{0}\right) \in \Xi$, and for all $t \geq t_{0},\|\xi(t)\| \leq k_{\mathrm{e}}\left\|\xi\left(t_{0}\right)\right\| \mathrm{e}^{-\lambda\left(t-t_{0}\right)}$.

\section{CLOSED-LOOP COMPENSATION}

We now consider how the estimation scheme from the previous section can be used to compensate for the perturbation in (2). Suppose that the control inputs available in the original system can be chosen to yield a system on the following form:

$$
\dot{x}=f(t, x)+B(t, x)(g(t, x, \theta)-g(t, x, \hat{\theta})) .
$$

Here, $v(t, x)$ in $(2)$ is equal to $-g(t, x, \hat{\theta})$. To state our result for this system, we need another assumption, concerning the behavior of the solutions of (13), both with and without perturbations.

Assumption 3. The function $f(t, x)$ is continuously differentiable on $\mathbb{R}_{\geq 0} \times \mathbb{R}^{n}$; the origin of the nominal system $\dot{x}=f(t, x)$ is UGAS; for any trajectory $\hat{\theta}(t) \in \Theta$, the solutions $x(t)$ of the perturbed system (13) are UGB; and for each compact $K$ there exists a class $\mathcal{K}$ function $\gamma$ such that for all $(t, x, \hat{\theta}) \in \mathbb{R}_{\geq 0} \times K \times \Theta$,

$$
\|B(t, x)(g(t, x, \theta)-g(t, x, \hat{\theta}))\| \leq \gamma(\|\tilde{\theta}\|) .
$$

Theorem 2. Suppose that Assumptions 1-3 hold. Then for each compact neighborhood $K^{\prime} \subset \mathbb{R}^{2 n}$ of the origin, there exist $k^{\prime}>0$ such that if $K_{\phi}$ is chosen such that $\lambda_{\min }\left(K_{\phi}\right)>k^{\prime}$, then the origin of (13), (12) is uniformly asymptotically stable with $K^{\prime} \times(\Theta-\Theta)$ contained in the region of attraction.

Proof. This proof is based on the proof of Panteley and Loría (2001, Lemma 2). The properties from Assumption 3 of the unperturbed system imply by Panteley and Loría (2001, Prop. 1) the existence of a Lyapunov function $V_{x}(t, x)$; class $\mathcal{K}_{\infty}$ functions $\alpha_{1}$ and $\alpha_{2}$; and a class $\mathcal{K}$ function $\alpha_{4}$ such that for all $(t, x) \in \mathbb{R}_{\geq 0} \times \mathbb{R}^{n}$,

$$
\begin{gathered}
\alpha_{1}(\|x\|) \leq V_{x}(t, x) \leq \alpha_{2}(\|x\|), \\
\frac{\partial V_{x}}{\partial t}(t, x)+\frac{\partial V_{x}}{\partial x}(t, x) f(t, x) \leq-V_{x}(t, x), \\
\left\|\frac{\partial V_{x}}{\partial x}(t, x)\right\| \leq \alpha_{4}(\|x\|) .
\end{gathered}
$$

Let $R>0$ be chosen large enough that $B:=\{(x, \xi) \mid$ $\|(x, \xi)\| \leq R\} \supset K^{\prime} \times(\Theta-\Theta)$. If $\left(x\left(t_{0}\right), \xi\left(t_{0}\right)\right) \in B$ and $\hat{\theta}\left(t_{0}\right) \in \Theta$, this implies that $\left\|x\left(t_{0}\right)\right\| \leq R$, and from the UGB property from Assumption 3, we therefore know that for all $t \geq t_{0}, x(t)$ is uniformly bounded. Let therefore $\lambda_{\min }\left(K_{\phi}\right)$ be chosen large enough to ensure exponential stability of the estimator according to Lemma 1.

By the exponential stability property of (12), we know that $\|\xi(t)\| \leq k_{\mathrm{e}}\left\|\xi\left(t_{0}\right)\right\| \mathrm{e}^{-\lambda\left(t-t_{0}\right)}$. Combining this with the UGB property of (13), we also know that for each $0<r \leq R$ there exists $c(r)>0$ such that if $\left\|\left(x\left(t_{0}\right), \xi\left(t_{0}\right)\right)\right\| \leq r$ and $\hat{\theta}\left(t_{0}\right) \in \Theta$, then $\|(x(t), \xi(t))\| \leq c(r)$.

Define $v(t)=V_{x}(t, x(t))$. We then have

$$
\dot{v}(t) \leq-v(t)+\alpha_{4}(c(r)) \beta\left(r, t-t_{0}\right),
$$

where $\beta\left(r, t-t_{0}\right):=\gamma\left(k_{\mathrm{e}} r \mathrm{e}^{-\lambda\left(t-t_{0}\right)}\right)$ is a class $\mathcal{K} \mathcal{L}$ function by Khalil (2002, Lemma 4.2). Let $\tau_{0} \geq t_{0}$. Multiplying by $\mathrm{e}^{t-\tau_{0}}$ on both sides and rearranging, we have for all $t \geq \tau_{0}$,

$$
\frac{\mathrm{d}}{\mathrm{d} t}\left(v(t) \mathrm{e}^{t-\tau_{0}}\right) \leq \alpha_{4}(c(r)) \beta\left(r, t-t_{0}\right) \mathrm{e}^{t-\tau_{0}} .
$$

Integrating from $\tau_{0}$ to $t$ on both sides and multiplying by $\mathrm{e}^{-\left(t-\tau_{0}\right)}$, we have

$$
\begin{aligned}
v(t) & \leq v\left(\tau_{0}\right) \mathrm{e}^{-\left(t-\tau_{0}\right)} \\
& +\alpha_{4}(c(r)) \int_{\tau_{0}}^{t} \mathrm{e}^{-(t-s)} \beta\left(r, s-t_{0}\right) \mathrm{d} s,
\end{aligned}
$$

which means that

$$
v(t) \leq v\left(t_{0}\right)+\alpha_{4}(c(r)) \beta(r, 0)\left(1-\mathrm{e}^{-\left(t-t_{0}\right)}\right) \leq \gamma^{\prime}(r),
$$

where $\gamma^{\prime}(r):=\alpha_{2}(r)+\alpha_{4}(c(r)) \beta(r, 0)$. Hence, $\|x(t)\| \leq$ $\alpha_{1}^{-1}\left(\gamma^{\prime}(r)\right)$, and $\alpha_{1}^{-1} \circ \gamma^{\prime}(r)$ is a class $\mathcal{K}_{\infty}$ function. Together with the expontential stability property of the origin of (12), this implies that the origin of the whole system (13), (12) is uniformly stable.

For some arbitrary $\varepsilon>0$, define $T_{1}$ large enough that that $\alpha_{4}(c(r)) \beta\left(r, T_{1}\right) \leq \varepsilon / 2$. From (15), we have for all $t \geq t_{0}+$ $T_{1}, v(t) \leq v\left(t_{0}+T_{1}\right) \mathrm{e}^{-\left(t-t_{0}-T_{1}\right)}+\frac{\varepsilon}{2}$. Let $T_{2} \geq T_{1}$ be chosen large enough that $\gamma^{\prime}(r) \mathrm{e}^{-\left(T_{2}-t_{0}-T_{1}\right)} \leq \varepsilon / 2$. Inserting the inequality $v(t) \leq \gamma^{\prime}(r)$, we then have, for all $t \geq t_{0}+T_{2}$, $v(t) \leq \gamma^{\prime}(r) \mathrm{e}^{-\left(T_{2}-t_{0}-T_{1}\right)}+\varepsilon / 2$, which furthermore implies $v(t) \leq \varepsilon$. Hence, for all $t \geq t_{0}+T_{2},\|x(t)\| \leq \alpha_{1}^{-1}(\varepsilon)$. Since 
$\alpha_{1}^{-1}(\varepsilon)$ can be chosen arbitrarily small by decreasing $\varepsilon$ and (12) is uniformly exponentially stable with $\Xi$ contained in the region of attraction, the whole system (13), (12) is therefore uniformly asymptotically stable with $K^{\prime} \times(\Theta-$ $\Theta)$ contained in the region of attraction.

\section{SIMULATION EXAMPLES}

Example 3. Consider the system

$$
\dot{x}=-x+\mathrm{e}^{\sin (t) \theta}+u,
$$

where $\theta \in\left[\theta_{\min }, \theta_{\max }\right]$ (i.e., $f(t, x)=f(x)=-x, B(t, x)=$ 1 , and $\left.g(t, x, \theta)=g(t, \theta)=\mathrm{e}^{\sin (t) \theta}\right)$. We wish to use $u$ to cancel the perturbation, and let $u=-\mathrm{e}^{\sin (t) \hat{\theta}}$. The first step is to design an update law to estimate $\theta$ from the full perturbation. As in Example 1, we use the mean value theorem to find that $\mathrm{e}^{\sin (t) \theta}-\mathrm{e}^{\sin (t) \hat{\theta}}=\sin (t) \mathrm{e}^{\sin (t) \bar{\theta}} \tilde{\theta}$, where $\bar{\theta}$ is a value between $\theta$ and $\hat{\theta}$. Hence, the choice of $M(t, x)=M(t)=\sin (t)$ satisfies (7) with $P(t, x)=$ $P(t)=\sin ^{2}(t) \mathrm{e}^{-\theta^{\prime}}$, where $\theta^{\prime}:=\max _{\theta \in \Theta}|\theta|$. We therefore let

$$
u_{\theta}(t, x, \hat{\phi}, \hat{\theta})=k_{\theta} \sin (t)\left(\hat{\phi}-\mathrm{e}^{\sin (t) \hat{\theta}}\right)+\text { p.t. }
$$

It is easily confirmed that the Lyapunov function (10) can be used in the same manner as in Example 2. We now check that the conditions of Assumption 2 hold. We have that

$$
d(t, x, \tilde{\theta})=\left(\theta \mathrm{e}^{\sin (t) \theta}-\hat{\theta} \mathrm{e}^{\sin (t) \hat{\theta}}\right) \cos (t) .
$$

Again using the mean value theorem, we find that $|d(t, x, \tilde{\theta})| \leq\left(1+\theta^{\prime}\right) \mathrm{e}^{\theta^{\prime}}|\tilde{\theta}|$. We also see that

$$
\left|u_{\theta}(t, x, \phi, \hat{\theta})-u(t, x, \hat{\phi}, \hat{\theta})\right|=k_{\theta}|\sin (t) \tilde{\phi}| \leq k_{\theta}|\tilde{\phi}|{ }^{1}
$$

Moving to Assumption 3, it is straightforward to see that the nominal, unperturbed system $\dot{x}=-x$ is UGAS and that the perturbed system is UGB (because $\theta$ and $\hat{\theta}$ are restricted to $\Theta)$. Finally, (14) holds with $\gamma(s)=\mathrm{e}^{\theta^{\prime}} s$.

Having confirmed that the assumptions hold, we implement the full estimator from (11). After canceling terms, this results in the following estimator:

$$
\begin{aligned}
\dot{z}= & -K_{\phi}\left(K_{\phi}+k_{\theta} \sin ^{2}(t) \mathrm{e}^{\sin (t) \hat{\theta}}-1\right) x \\
& \quad-\left(K_{\phi}+k_{\theta} \sin ^{2}(t) \mathrm{e}^{\sin (t) \hat{\theta}}\right) z-\sin (t) \mathrm{e}^{\sin (t) \hat{\theta}}(\text { p.t. }), \\
\dot{\hat{\theta}}= & k_{\theta} \sin (t)\left(z+K_{\phi} x\right)+\text { p.t. }
\end{aligned}
$$

We simulate this system with $\theta_{\max }=-10$ and $\theta_{\max }=$ 10, letting $\theta$ vary in steps between -2 and 4 to get an impression of the response. We use the estimator parameters $K_{\phi}=10, k_{\theta}=3$. The results can be seen in Figure 1, where, for the sake of comparison, we have also plotted the response using a gradient algorithm (i.e., $\left.\dot{\hat{\theta}}=\Gamma \sin (t) \mathrm{e}^{\sin (t) \hat{\theta}} x\right)$ with gain $\Gamma=1$. Noise has been added to the measurement of the state $x$ used in both algorithms. The noise is added with sample time 0.001 , and has variance 1 . The parameter projection is not active at any point in the simulation.

\footnotetext{
1 In checking the condition on $u_{\theta}$ from Assumption 2, we have not included any consideration of the projection term. This term does not destroy the property of $u_{\theta}$ in question; however, the proof of this is omitted due to space constraints.
}

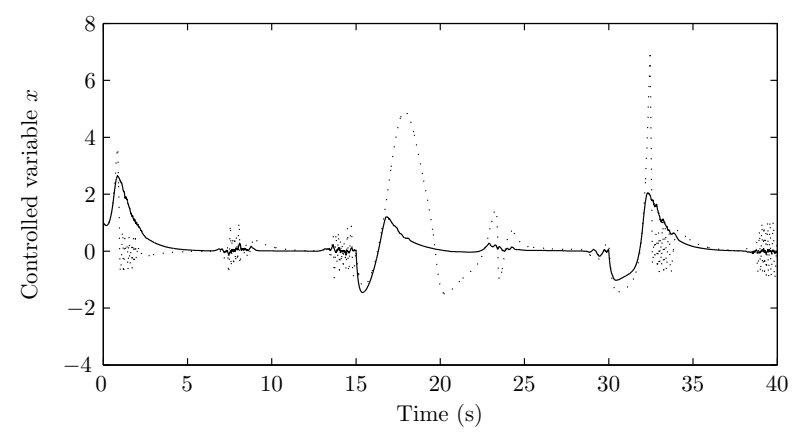

(a) Controlled variable, nonlinear method (solid), and gradient method (dotted)

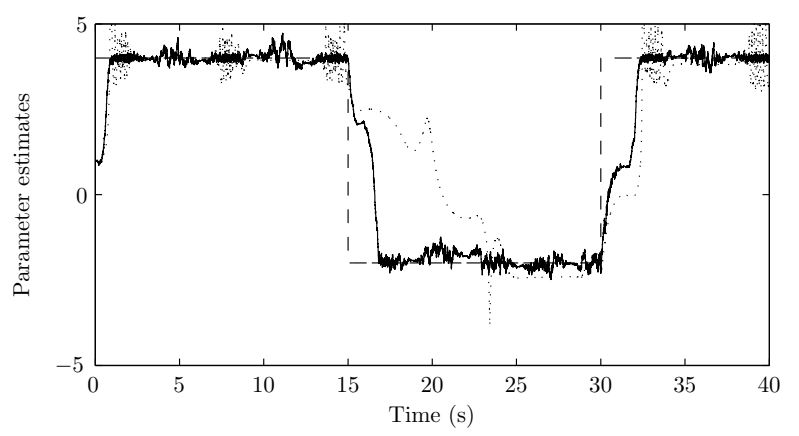

(b) Unknown parameter (dashed), estimate with nonlinear method (solid), and estimate with gradient method (dotted)

Fig. 1. Simulation results for example 3

Example 4. In our final example, we consider the problem of estimating the unknown parameters of a deadzone nonlinearity. Consider the system

$$
\dot{x}=-x^{3}+\mathrm{dz}(\delta),
$$

where $\delta$ represents some known time-varying input to the equation and $\mathrm{dz}(\cdot)$ is a deadzone nonlinearity described by

$$
\mathrm{dz}(\delta)= \begin{cases}m_{l} \delta+m_{l} b_{l}, & \delta<-b_{l} \\ 0 & -b_{l} \leq \delta \leq b_{r} \\ m_{r} \delta-m_{r} b_{r}, & \delta>b_{r}\end{cases}
$$

The positive constants $m_{l}$ and $m_{r}$ are unknown slopes in the left and right linear regions of the deadzone, and the positive constants $b_{l}$ and $b_{r}$ represent unknown break points for the deadzone. We will estimate the constants $m_{l}, m_{r}, b_{l}^{\prime}:=m_{l} b_{l}$ and $b_{r}^{\prime}:=m_{r} b_{r}$ (from which $b_{l}=b_{l}^{\prime} / m_{l}$ and $b_{r}=b_{r}^{\prime} / m_{r}$ can be calculated) and define the vector $\theta:=\left[m_{l}, b_{l}^{\prime}, m_{r}, b_{r}^{\prime}\right]^{\top}$. As before, the main problem is finding an update law for estimating the parameters if the full perturbation $\phi=\mathrm{dz}(\delta)$ is known. To solve this problem, we first suppose that we know that $\delta<-b_{l}$, i.e., that $\delta$ is in the left linear region of the deadzone. The problem then reduces to estimating a bias $\left(b_{l}^{\prime}\right)$ and a scaling $\left(m_{l}\right)$. In this case, the choice of

$$
\dot{\hat{m}}_{l}=k_{\theta_{1}} \delta\left(\phi-\hat{m}_{l} \delta-\hat{b}_{l}^{\prime}\right), \quad \dot{\hat{b}}_{l}^{\prime}=k_{\theta_{2}}\left(\phi-\hat{m}_{l} \delta-\hat{b}_{l}^{\prime}\right)
$$

leads to the error dynamics

$$
\begin{gathered}
\dot{\tilde{m}}_{l}=-k_{\theta_{1}} \delta\left(\phi-\hat{m}_{l} \delta-\hat{b}_{l}^{\prime}\right)=-k_{\theta_{1}} \delta\left(\tilde{m}_{l} \delta+\tilde{b}_{l}^{\prime}\right), \\
\dot{\tilde{b}}_{l}^{\prime}=-k_{\theta_{2}}\left(\phi-\hat{m}_{l} \delta-\hat{b}_{l}^{\prime}\right)=-k_{\theta_{2}}\left(\tilde{m}_{l} \delta+\tilde{b}_{l}^{\prime}\right) .
\end{gathered}
$$

(We will later replace $\phi$ by $\hat{\phi}$.) For this second-order system, we apply a slight variation of the LFC from (10):

$$
V_{\mathrm{u}}\left(t, \tilde{\theta}_{1,2}\right)=\frac{1}{2} \tilde{\theta}_{1,2}^{\mathrm{T}} K_{\theta}^{-1} \tilde{\theta}_{1,2}-\mu \tilde{\theta}_{1,2}^{\mathrm{T}} \int_{t}^{\infty} \mathrm{e}^{t-\tau} P(\tau) \mathrm{d} \tau \tilde{\theta}_{1,2},
$$


where $\tilde{\theta}_{1,2}=\left[\tilde{m}_{l}, \tilde{b}_{l}^{\prime}\right], K_{\theta}=\operatorname{diag}\left(k_{\theta_{1}}, k_{\theta_{2}}\right)$ and $P(t)=$ $\left[\begin{array}{cc}\delta^{2} & \delta \\ \delta & 1\end{array}\right]$. We suppose that $\delta$ is uniformly bounded and that (9) holds. This is a standard persistency of excitation condition, requiring variation in $\delta$ in the left linear region of the deadzone. The time derivative of the LFC is then

$$
\begin{aligned}
\dot{V}_{\mathrm{u}}\left(t, \tilde{\theta}_{1,2}\right)= & -(1-\mu)\left(\delta \tilde{m}_{l}+\tilde{b}_{l}^{\prime}\right)^{2} \\
& -\mu \tilde{\theta}_{1,2}^{\top} \int_{t}^{\infty} \mathrm{e}^{t-\tau} P(\tau) \mathrm{d} \tau \tilde{\theta}_{1,2} \\
& +2 \mu \tilde{\theta}_{1,2}^{\top} \int_{t}^{\infty} \mathrm{e}^{t-\tau} P(\tau) \mathrm{d} \tau K_{\theta}[\delta, 1]^{\top}\left(\delta \tilde{m}_{l}+\tilde{b}_{l}^{\prime}\right) \\
\leq & -(1-\mu)\left|\delta \tilde{m}_{l}+\tilde{b}_{l}\right|^{2}-\mu \mathrm{e}^{-T} \varepsilon\left\|\tilde{\theta}_{1,2}\right\|^{2} \\
& +2 \mu M_{P} M_{M}\left\|K_{\theta}\right\|\left\|\tilde{\theta}_{1,2}\right\| \delta \tilde{m}_{l}+\tilde{b}_{l} \mid,
\end{aligned}
$$

where $M_{P}$ and $M_{M}$ are bounds on $\|P(t)\|$ and $\left\|[\delta, 1]^{\top}\right\|$. The last expression is a quadratic expression, which is negative definite provided $\mu$ is chosen sufficiently small.

We now have an update law and a Lyapunov function in the case when $\delta$ is in the left linear region of the deadzone. Similarly, we can design an update law and Lyapunov function for the case when $\delta$ is in the right linear region of the deadzone. Noting that $\phi<0$ implies that $\delta$ is in the left linear region and $\phi>0$ implies that it is in the right linear region, we now take these the two separate update laws and create a complete one as follows:

$$
\begin{aligned}
\dot{\hat{m}}_{l} & =(\phi<0) k_{\theta_{1}} \delta\left(\phi-\hat{m}_{l} \delta-\hat{b}_{l}^{\prime}\right)+\text { p.t. } ._{1}, \\
\dot{\hat{b}}_{l}^{\prime} & =(\phi<0) k_{\theta_{2}}\left(\phi-\hat{m}_{l} \delta-\hat{b}_{l}^{\prime}\right)+\text { p.t. } \\
\dot{\hat{m}}_{r} & =(\phi>0) k_{\theta_{3}} \delta\left(\phi-\hat{m}_{r} \delta+\hat{b}_{r}^{\prime}\right)+\text { p.t. } 3, \\
\dot{\hat{b}}_{r}^{\prime} & =-(\phi>0) k_{\theta_{4}}\left(\phi-\hat{m}_{r} \delta+\hat{b}_{r}^{\prime}\right)+\text { p.t. } \cdot_{4},
\end{aligned}
$$

where $(\phi<0)$ and $(\phi>0)$ are logical expressions evaluating to 1 (true) or 0 (false). A Lyapunov function is formed by taking the sum of the two separate Lyapunov functions found for the left and right linear regions, where $P(\tau)$ is multiplied by $(\phi<0)$ in the Lyapunov function for the left linear region and by $(\phi>0)$ for the right linear region. The resulting persistency of excitation condition, ensuring exponential stability of the full error dynamics requires variation in $\delta$, in both the left and right linear regions. The full estimator is implemented following (11), using the above update law (where $\phi$ is replaced by $\hat{\phi}$ ).

In checking the conditions of Assumption 2, we see that the discontinuity of the logical expressions $(\phi<0)$ and $(\phi>0)$ creates a problem with the second condition. This is circumvented without altering the basic stability results by replacing all occurences of the expressions with $-\frac{2}{\pi} \min \{0, \arctan (\phi / \varepsilon)\}$ and $\frac{2}{\pi} \max \{0, \arctan (\phi / \varepsilon)\}$, where $\varepsilon$ is some small, positive number. Another problem is that the deadzone nonlinearity is not differentiable at the breakpoints, which means that $d(t, x, \tilde{\theta})$ is not always well-defined and creates a problem with the first condition of Assumption 2. This effectively creates a disturbance which we choose to ignore, and justify this by noting that the deadzone nonlinearity can be approximated by a smooth function. We implement the full estimator and simulate the system letting $\delta=5 \sin (t), m_{l}=1.15, b_{l}=$ $0.2, m_{r}=0.85$ and $b_{r}=0.3$. We use the constants $K_{\phi}=10$ and $k_{\theta_{1}}=k_{\theta_{3}}=0.2, k_{\theta_{2}}=k_{\theta_{4}}=0.1$, and $\varepsilon=0.1$. The results can be seen in Figure 2. It is clear that the parameters converge to their correct values, although this

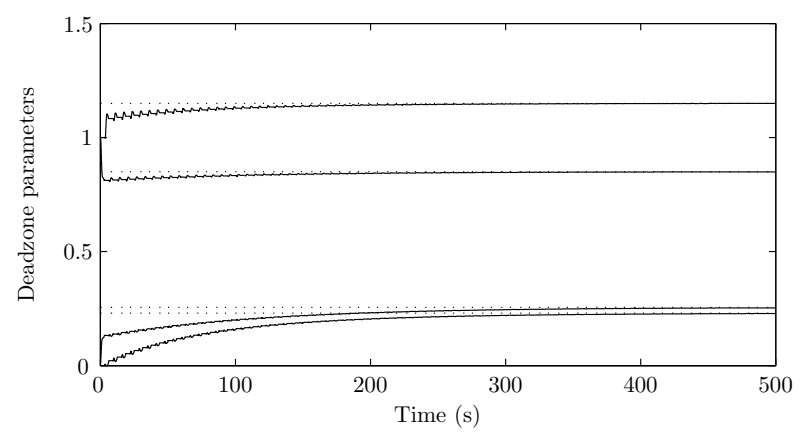

Fig. 2. Simulation results for Example 4. Solid lines: estimates; dotted lines: actual parameters

takes a considerable amount of time. This is a consequence of estimating four variables from one signal.

If $\delta$ represents a control signal, it is possible to compensate for the deadzone nonlinearity by using a deadzone inverse. Such compensation with adaptation of deadzone parameters has previously been investigated (e.g., Recker and Kokotović (1991); Tao and Kokotović (1994)).

\section{REFERENCES}

A. M. Annaswamy, F. P. Skantze, and A.-P. Loh. Adaptive control of continuous time systems with convex/concave parametrization. Automatica, 34(1):33-49, 1998.

J. D. Bošković. Stable adaptive control of a class of first-order nonlinearly parameterized plants. IEEE Trans. Automat. Contr., 40(2):347-350, 1995.

J. D. Bošković. Adaptive control of a class of nonlinearly parameterized plants. IEEE Trans. Automat. Contr., 43(7):930-934, 1998.

A. Chakrabortty and M. Arcak. A two-time scale redesign for robust stabilization and performance recovery of uncertain nonlinear systems. In Proc. American Contr. Conf., New York, NY, 2007.

V. Fomin, A. Fradkov, and V. Yakubovich, editors. Adaptive Control of Dynamical Systems. Nauka, Moskow, 1981.

P. A. Ioannou and J. Sun. Robust Adaptive Control. Prentice Hall, Upper Saddle River, NJ, 1996.

H. K. Khalil. Nonlinear Systems. Prentice Hall, Upper Saddle River, NJ, third edition, 2002.

M. Krstić, I. Kanellakopoulos, and P. Kokotović. Nonlinear and Adaptive Control Design. Wiley, New York, 1995.

A.-P. Loh, A. M. Annaswamy, and F. P. Skantze. Adaptation in the presence of a general nonlinear parameterization: An error model approach. IEEE Trans. Automat. Contr., 44(9):1634-1652, 1999.

R. Ortega. Some remarks on adaptive neuro-fuzzy systems. Int. J. Adapt. Contr. Signal Process., 10:79-83, 1996.

E. Panteley and A. Loría. Growth rate conditions for uniform asymptotic stability of cascaded time-varying systems. Automatica, 37 (3):453-460, 2001.

Z. Qu. Adaptive and robust controls of uncertain systems with nonlinear parameterization. IEEE Trans. Automat. Contr., 48 (10):1817-1823, 2003.

Z. Qu, R. A. Hull, and J. Wang. Globally stabilizing adaptive control design for nonlinearly-parametrized systems. IEEE Trans. Automat. Contr., 51(6):1073-1079, 2006.

D. A. Recker and P. V. Kokotović. Adaptive nonlinear control of systems containing a deadzone. In Proc. 30th IEEE Conf. Dec. Contr., Brighton, England, 1991.

G. Tao and P. V. Kokotović. Adaptive control of plants with unknown dead-zones. IEEE Trans. Automat. Contr., 39(1):5968, 1994.

T. Zhang, S. S. Ge, C. C. Hang, and T. Y. Chai. Adaptive control of first-order systems with nonlinear parameterization. IEEE Trans. Automat. Contr., 45(8):1512-1516, 2000. 